\title{
A cmap-enabled gene expression signature-matching approach identifies small-molecule inducers of accelerated cell senescence
}

\author{
Ding $\mathrm{Wu}^{1}$, Brett Pepowski ${ }^{1}$, Satoe Takahashi ${ }^{2}$ and Stephen J. Kron ${ }^{*}$
}

\begin{abstract}
Background: Diverse stresses including genotoxic therapy can induce proliferating cancer cells to undergo cellular senescence and take on the characteristic phenotypes of replicative cellular aging. This accelerated or therapyinduced senescence has been alternatively proposed to contribute to therapeutic efficacy or resistance. Toward better understanding this cell state, we sought to define the core transcriptome of accelerated senescence in cancer cells.

Results: We examined senescence induced by ionizing irradiation or ectopic overexpression of the stoichiometric

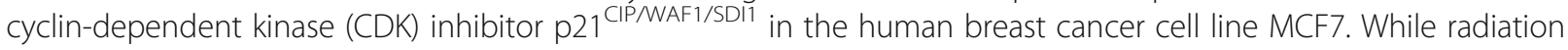
produces a strong DNA damage response, ectopic expression of p21 arrests cell cycle progression independently of DNA damage. Both conditions promoted senescence within 5 days. Microarray analysis revealed 378 up- and 391 down-regulated genes that were shared between the two conditions, representing a candidate signature. Systems analysis of the shared differentially expressed genes (DEGs) revealed strong signals for cell cycle control and DNA damage response pathways and predicted multiple upstream regulators previously linked to senescence. Querying the shared DEGs against the Connectivity Map (cmap) database of transcriptional responses to small molecules yielded 20 compounds that induce a similar gene expression pattern in MCF7 cells. Of 16 agents evaluated, six induced senescence on their own. Of these, the selective estrogen receptor degrader fulvestrant and the histone acetyltransferase inhibitor vorinostat did so without causing chromosomal damage.
\end{abstract}

Conclusions: Using a systems biology approach with experimental validation, we have defined a core gene expression signature for therapy-induced senescence.

Keywords: Radiation-induced senescence, p21-induced senescence, Transcriptome analysis, Differential expression genes, DNA damage foci, Upstream regulator factors, Connectivity map analysis, Small molecule inhibitors

\section{Background}

From its initial observation by Hayflick and Moorhead [1], the limited proliferative capacity of normal cells in culture and their terminal arrest via replicative senescence has been considered the cellular equivalent of organismal aging. Replicative senescence (RS) has long been attributed to telomere erosion but may also reflect mitochondrial and

\footnotetext{
* Correspondence: skron@uchicago.edu

'Department of Molecular Genetics and Cell Biology and Ludwig Center for Metastasis Research, The University of Chicago, 929 East 57th Street, GCIS W522A, Chicago, IL 60637, USA

Full list of author information is available at the end of the article
}

chromosomal replication stress and other effects of accumulated oxidative damage [2]. Stress-induced premature senescence (SIPS) can be triggered by genotoxic stress, oxidative stress, oncogenic stress, chromatin disruption and other acute stresses [3-6]. Therapy-induced senescence (TIS) is observed in normal and tumor cells following radiation and/or chemotherapy. Programmed senescence is a recently described feature of early development [7]. Common features of these diverse forms of senescence include enlarged cell size, unrepaired genomic damage, proliferative arrest linked to expression of the $\mathrm{p} 21^{\mathrm{CIP} / \mathrm{WAF} 1}$

(c) The Author(s). 2019 Open Access This article is distributed under the terms of the Creative Commons Attribution 4.0 International License (http://creativecommons.org/licenses/by/4.0/), which permits unrestricted use, distribution, and reproduction in any medium, provided you give appropriate credit to the original author(s) and the source, provide a link to the Creative Commons license, and indicate if changes were made. The Creative Commons Public Domain Dedication waiver (http://creativecommons.org/publicdomain/zero/1.0/) applies to the data made available in this article, unless otherwise stated. 
and/or $\mathrm{p} 16^{\text {Ink4A }}$ stoichiometric cyclin-dependent kinase inhibitors, and activation of senescence-associated $\beta$-galactosidase activity (SA- $\beta$-Gal) $[8-12]$.

Current understanding is that a common initiating event for senescence is accumulation of unrepairable DNA damage such as eroded telomeres, collapsed replication forks or chromosomal double strand breaks (DSBs). This places senescence as an endpoint of the DNA damage checkpoint pathway, where p53-dependent p21 expression initiates cell cycle arrest. Nonetheless, what directs cells toward senescence rather than cell death, autophagy or a return to proliferation remains poorly understood. Once formed, senescent cells can persist indefinitely and actively perform autocrine and paracrine signaling, which has been implicated in inflammatory responses, fibrosis, carcinogenesis and other changes in proliferation and gene expression. This senescence-associated secretory phenotype (SASP) appears to depend on DNA damage-induced, NF-kBdependent expression of inflammatory cytokines, chemokines, and growth factors [13]. Separating the senescence and SASP pathways, ectopic expression of p21 or p16 can induce senescence without DNA damage or a SASP [14] and targeting NF- $\mathrm{kB}$, PARP, HMG-CoA reductase, p38, JAK, MLL1, BRD4 or other targets can suppress the SASP without blocking senescence after DNA damage [15-18]. Senescence itself can be blocked by protecting telomeres from erosion, limiting other DNA damage, mitigating oxidative stress or disrupting downstream signaling [19, 20]. There is a complementary interest in restoring replicative senescence in cancer by blocking telomere maintenance or promoting therapy-induced senescence after genotoxic cancer therapy [21,22]. This has stimulated a recent focus by our group and others toward identifying agents that promote cancer cell senescence on their own or in combination with other genotoxic stresses [23, 24].

A weakness shared by studies to date is that while pathways upstream of the SASP are now well-defined, the mechanism of senescence itself remains obscure, meaning that specific targets have yet to be identified. We reasoned that along with a characteristic cellular phenotype, senescent cells may display a distinctive gene expression signature independent of whether the SASP is expressed or not. Thus, we have compared the transcriptomes of senescent MCF7 human mammary carcinoma cells formed by radiation or overexpression of the p21 protein to generate a candidate senescence signature. Systems analysis flagged mostly pathways associated with cell cycle arrest, offering minimal insight, although several upstream signaling regulators were identified that may determine specific features of senescent cell arrest. Toward identifying new chemical probes for senescence, we queried the Connectivity Map (cmap) database of microarray expression data from cell lines treated with bioactive small molecules [25]. Of the
20 highest scoring gene expression patterns, we examined 16 compounds in vitro and identified four that promote senescence along with DNA damage foci and two others that appear to phenocopy p21 overexpression by inducing senescence without DNA damage foci. This work provides a pathway toward defining a core gene expression signature for senescence and leveraging this pattern to discover candidate chemical probes to dissect critical pathways.

\section{Methods}

\section{Cell lines and cell culture}

Both cell lines $\mathrm{MCF}^{\mathrm{GFP}-\mathrm{IBD}}$ and $\mathrm{MCF}^{\mathrm{p} 21}$ were generated in our laboratory through modification of the MCF7 Tet-On Advanced cell line (Clontech). The generation and characterization of the $\mathrm{MCF}^{\mathrm{GFP}-\mathrm{IBD}}$ cell line has been described [26]. Briefly, GFP fused to the human 53BP1 ionizing-radiation induced foci binding domain (IBD) was cloned and transduced into the MCF7 Tet-On Advanced cell line (Clontech). MCF7 ${ }^{\mathrm{p} 21}$ was developed from MCF7FUCCI cells into which an inducible p21 construct was transduced. Briefly, MCF7-FUCCI cells were generated by lentiviral transduction of MCF7 Tet-On cells with FUCCI constructs [27] followed by cell sorting. To express p21 in MCF7-FUCCI, the wild-type (WT) CDKN1A open reading frame (p21, Origene NM_078467) [28] from human cDNA was PCR amplified with primers 5'CGACGGATCCATGT CAGAACCGGCTGGGGATGTCCGTCAG3' and 5'CGA CGAATTCTTAGGGCTTCCTCTTGGAGAAGATCAGC CG3'. PCR products were then digested with BamHI and EcoRI, and cloned into pLVX-Tight-Puro (Clontech) to obtain pLVX-p21. pLVX-p21 lentivirus was prepared using packaging mix (Clontech) and transduced into MCF7FUCCI Tet-On Advanced, followed by puromycin selection at $0.5 \mu \mathrm{g} / \mathrm{ml}$ to obtain $\mathrm{MCF}^{\mathrm{p} 21}$ cells.

Cell lines were maintained in Dulbecco's Modified Eagle Medium (DMEM) with $4 \mathrm{mML}$-glutamine (Invitrogen), supplemented with $10 \%$ Tet system approved FBS (Clontech) and penicillin-streptomycin (Pen-Strep, Thermo) at $5 \%$ carbon dioxide and $37^{\circ} \mathrm{C} \cdot \mathrm{MCF}^{\mathrm{p} 21}$ and MCF7-FUCCI cells were maintained with $0.5 \mu \mathrm{g} / \mathrm{ml}$ puromycin.

\section{Immunofluorescence and IR-induced foci (IRIF) imaging}

For immunofluorescence, cells were fixed with $2 \%$ paraformaldehyde (PFA) and stained with antiphospho- $\gamma \mathrm{H} 2 \mathrm{AX}$ (Millipore, clone JBW301) detected with anti-mouse Alexa 488 (Jackson) or anti-p21 (Thermo) detected with anti-rabbit Alexa 647 (Jackson). Images were captured on a Zeiss Axiovert $40 \mathrm{CFL}$ microscope with a 40X Plan-Neofluar objective and Axiocam digital camera controlled by AxioVision 4.8 software. Images were pseudo-colored in Adobe Photoshop or ImageJ (http://imagej.nih.gov/ij/). For IRIF 
imaging, $\quad \mathrm{MCF} 7^{\mathrm{GFP}-\mathrm{IBD}}$ was induced with $1 \mu \mathrm{g} / \mathrm{ml}$ doxycycline (Sigma) for $24 \mathrm{~h}$. On the second day cells were irradiated with 6 Gy of ionizing radiation (IR) or treated with compounds. Cells were fixed with $2 \%$ PFA and foci were imaged as previously described [19] .

\section{SA- $\beta$-Gal senescence assay}

The SA- $\beta$-Gal assay was performed as described previously [12] with minor modification [19]. Briefly, cells were seeded on microscope cover slips in 24-well plates at $1 \times 10^{4}$ per well. After 1 day, cells were treated with $10 \mu \mathrm{M}$ compounds and cultured for 5 more days. On the sixth day, cells were fixed with $2 \%$ PFA for 5 min and washed with PBS. Then the cells were incubated at $37^{\circ} \mathrm{C}$ (without $\mathrm{CO}_{2}$ ) in staining solution consisting of $1 \mathrm{mg} / \mathrm{ml} \mathrm{X}$-gal (5-bromo-4-chloro-3-indolyl $\beta$-D-galactopyranoside) in $40 \mathrm{mM}$ citric acid, $\mathrm{pH}$ 6.0, $3.3 \mathrm{mM}$ potassium ferrocyanide, $3.3 \mathrm{mM}$ potassium ferricyanide, $150 \mathrm{mM} \mathrm{NaCl}$ and 20 $\mathrm{mM} \mathrm{MgCl}$. Staining lasted for 4-8 h, until color became evident. Images were captured on a Zeiss Axiovert $200 \mathrm{M}$ microscope with 20X Plan-NeoFluar objective and Axiocam digital color camera controlled by AxioVision Rel 4.8 software.

\section{RNA extraction, Illumina array hybridization and transcriptome analysis}

RNA was extracted using Trizol (Invitrogen) and further purified using RNeasy mini kits (Qiagen). $1 \mu \mathrm{g}$ total RNA was used for generation of cRNA which was hybridized to Illumina Microarray HumanHT12. All cRNA probes, oligonucleotide microarray manipulations, and scanning of arrays were carried out by the University of Chicago Functional Genomics core facility (https://fgf.uchicago.edu/). Data were analyzed by Illumina GenomeStudio (background correction, quantile normalization) and filtered by $p \leq 0.05$. Differentially expressed genes were detected with Significance Analysis of Microarrays (SAM) [29] with a 1.5 fold cutoff and false discovery rate of 0.01. A Python program was written to collapse genes based on average of probes (Additional file 1).

\section{Gene Expression Pathway analysis}

Ingenuity Pathway Analysis (IPA, https://www.qiagenbioinformatics.com/products/ingenuity-pathway-analysis/) was applied for functional pathway analysis. Canonical Pathway Analysis identified the pathways from the Ingenuity Pathways Analysis library of canonical pathways that were significantly enriched. The significance of the association between the uploaded dataset and the canonical pathway was measured in ratio and $p$-value from Fisher's exact test. In Database for Annotation, Visualization and Integrated Discovery (DAVID) (https:// david-d.ncifcrf.gov/) [30] Kyoto Enrichment of Genes and
Genomics (KEGG) [31] pathways analysis was applied. Enriched Gene Ontology (GO) terms in cellular process were analyzed through GOrilla (http://cbl-gorilla.cs.technion.ac.il/) [32] and REVIGO (http://revigo.irb.hr/) [33].

\section{Connectivity Map analysis}

Connectivity Map build02 [25] was used, containing more than 7000 expression profiles, representing 1309 small molecules, available at http://www.broad.mit.edu/ $\mathrm{cmap} /$. Since cmap uses the Affymetrix platform, we first changed the gene list from gene symbol to Affymetrix probe through Affymetrix batch query. Then the obtained probe list as a query signature (include two lists consisting of $\sim 500$ up-regulated gene probes and $\sim 400$ down-regulated gene probes) was used to run against the cmap database to generate hits. In cmap, a "perturbagen" is defined as any small molecule or genetic reagent. The similarity between the gene expression profile of the query signature and that of a cmap instance is measured by the connectivity score, ranging from -1 to 1 . A nonparametric, rank-based pattern matching strategy based on Kolmogorov-Smirnov statistics was used to determine connectivity score and $p$-value.

\section{Results}

\section{Radiation-induced and p21-mediated senescence}

In this study, we reexamined accelerated senescence triggered by ionizing radiation (IR) compared to ectopic expression of $\mathrm{p} 21$. We used MCF7 ${ }^{\mathrm{GFP}-\mathrm{IBD}}$ cells [26] expressing GFP fused to the 53BP1 binding domain (GFPIBD), to track ionizing radiation induced foci (IRIF) formation at DNA double strand breaks (DSBs). Along with the GFP-IBD foci, we also used immunofluorescence detection of phospho- $\mathrm{H} 2 \mathrm{AX}(\gamma \mathrm{H} 2 \mathrm{AX})$ as a second DSB reporter. To study senescence triggered by ectopic expression of $\mathrm{p} 21$, we constructed $\mathrm{MCF} \mathrm{7}^{\mathrm{p} 21}$ by transduction of MCF7 cells with a lentivirus encoding a Tet-On regulated CDKN1A (p21) gene. To induce senescence, MCF7 ${ }^{\text {GFP-IBD }}$ cells were irradiated with 6 Gy and MCF7 ${ }^{\mathrm{p} 21}$ cells were treated with $1 \mu \mathrm{g} / \mathrm{ml}$ doxycycline. After 5 days, both cell lines adopted characteristic features of senescent cells including enlarged cell and nuclear size, a flattened morphology, and increased SA- $\beta$-Gal staining compared to untreated controls (Fig. 1a). As expected, only the irradiated cells displayed increased $\gamma \mathrm{H} 2 \mathrm{AX}$ foci while cells overexpressing p21 showed no similar effects (Fig. 1b). Immunofluorescence with anti-p21 revealed increased nuclear expression in both the irradiated MCF7 ${ }^{\mathrm{GFP}-\mathrm{IBD}}$ and doxycycline-induced MCF7 ${ }^{\mathrm{p} 21}$ cells at 5 days (Fig. 1c).

\section{Functional gene expression studie}

Toward identifying a shared pattern of differentially expressed genes (DEGs) between radiation-induced and 


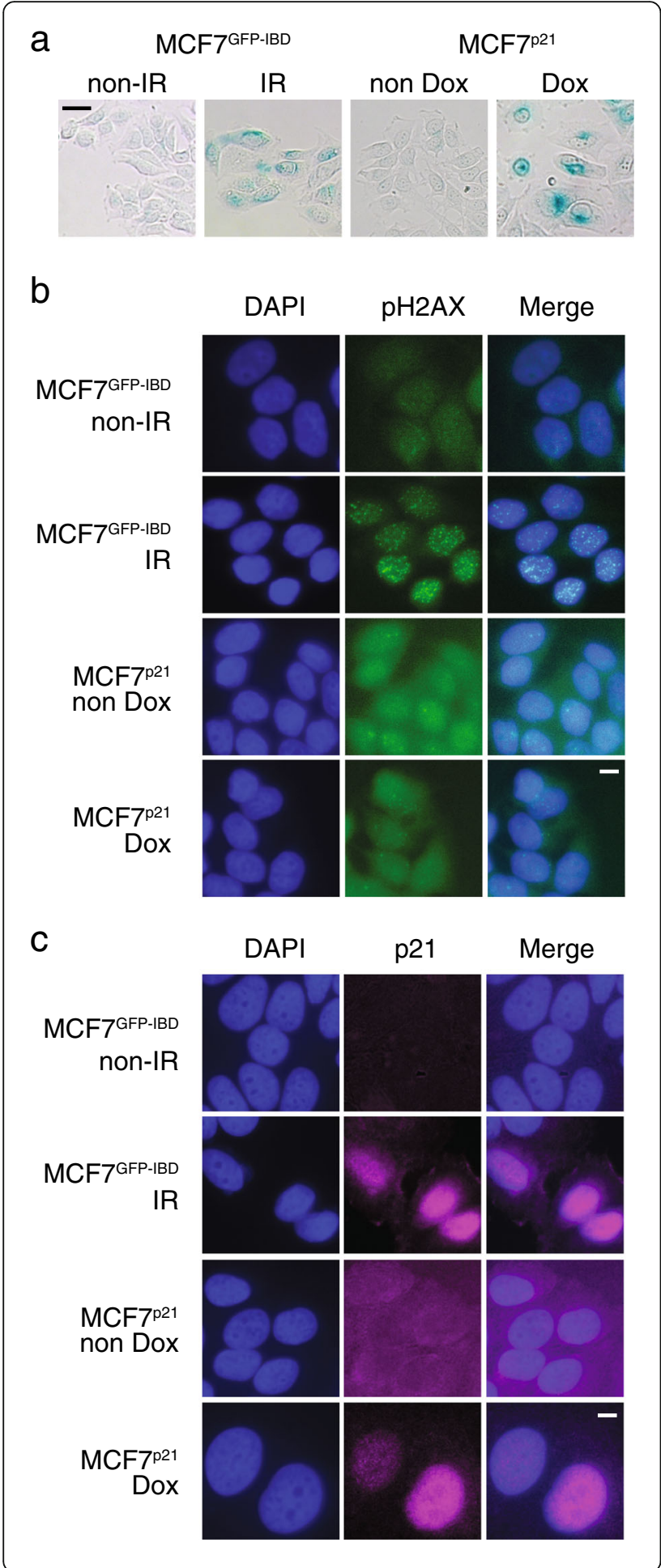

Fig. 1 Radiation and p21 overexpression induce similar senescence phenotypes. a SA- $\beta$-gal assay of MCF7 ${ }^{\text {GFP-IBD }}$ cells, 5 days after 6 Gy irradiation, compared to MCF $7^{\mathrm{p} 21}$ cells, 5 days after induction with doxycycline, displays activation of beta-galactosidase activity (blue staining) under both conditions. Scale bar $=20 \mu \mathrm{m}$. b

Immunofluorescence staining for phospho-H2AX in MCF7 ${ }^{\text {GFP-IBD }}$ cells demonstrates nuclear foci $3 \mathrm{~h}$ after 6 Gy irradiation while MCF7 ${ }^{\mathrm{p} 21}$ cells display no foci $3 \mathrm{~h}$ after induction with doxycycline. Scale bar $=$ 20 um. c Immunofluorescence staining for p21 in MCF $7^{\text {GFP-IBD }}$ cells, 5 days after 6 Gy irradiation, compared to MCF ${ }^{\text {p21 }}$ cells, 5 days after induction with doxycycline, demonstrates increased expression under each condition. Untreated controls are shown for each assay. Scale bar $=20 \mu \mathrm{m}$.

p21-mediated senescence, we performed microarray transcriptome analysis. RNA was obtained from irradiated $\mathrm{MCF}^{\mathrm{GFP}-\mathrm{IBD}}$ and doxycycline-induced $\mathrm{MCF}^{\mathrm{P} 21}$ cells after 5 days incubation along with untreated controls. Samples were reverse transcribed, fluorescently labeled, combined in pairs to their respective controls, hybridized to Illumina Human HT12 chips and analyzed with GenomeStudio. The radiation-induced senescent cells yielded 15,964 transcript probes at a detection $p$-value less than 0.05 while the p21-induced sample yielded 15,936 probes. SAM analysis to identify differentially expressed genes at $\mathrm{FDR}=0.01$ and fold change $\geq 1.5$ identified 1976 transcript probes in radiation senescent cells and 1819 in p21 senescent cells. Averaging fold expression between multiple probes identifying a common gene resulted in 1701 DEGs for the radiation sample and 1544 for the p21 sample, of which 790 were shared. Plotting the fold expression in radiation- versus p21-induced senescence of all genes showed many genes that displayed similar differential regulation (Fig. 2). Among common DEGs, 769 of 790 (97\%) showed the same direction of change, with 391 downregulated and 378 upregulated (Fig. 3, Additional file 2), consistent with the expression pattern of components of a senescence signature. Toward systems level analysis, the radiation-induced DEGs, p21-induced DEGs and the 769 shared DEGs were subjected to Ingenuity Pathway Analysis (IPA, https://www.qiagenbioinformatics.com/ products/ingenuity-pathway-analysis/), KEGG pathways study in Database for Annotation, Visualization and Integrated Discovery (DAVID) [31] and visualization in REVIGO [33].

IPA analysis of the shared DEGs yielded 12 canonical pathways at a cut-off $\log (p$-value) of 2 ( $p$-value $\leq 0.03$, Table 1), with several related to DNA damage response and cell cycle, including Role of BRCA1 in DNA Damage Response, Role of CHK Proteins in Cell Cycle Checkpoint Control, Mitotic Roles of Polo-Like Kinase, and Cell Cycle: G2/M DNA Damage Checkpoint Regulation. Among genes that flagged these pathways were multiple 


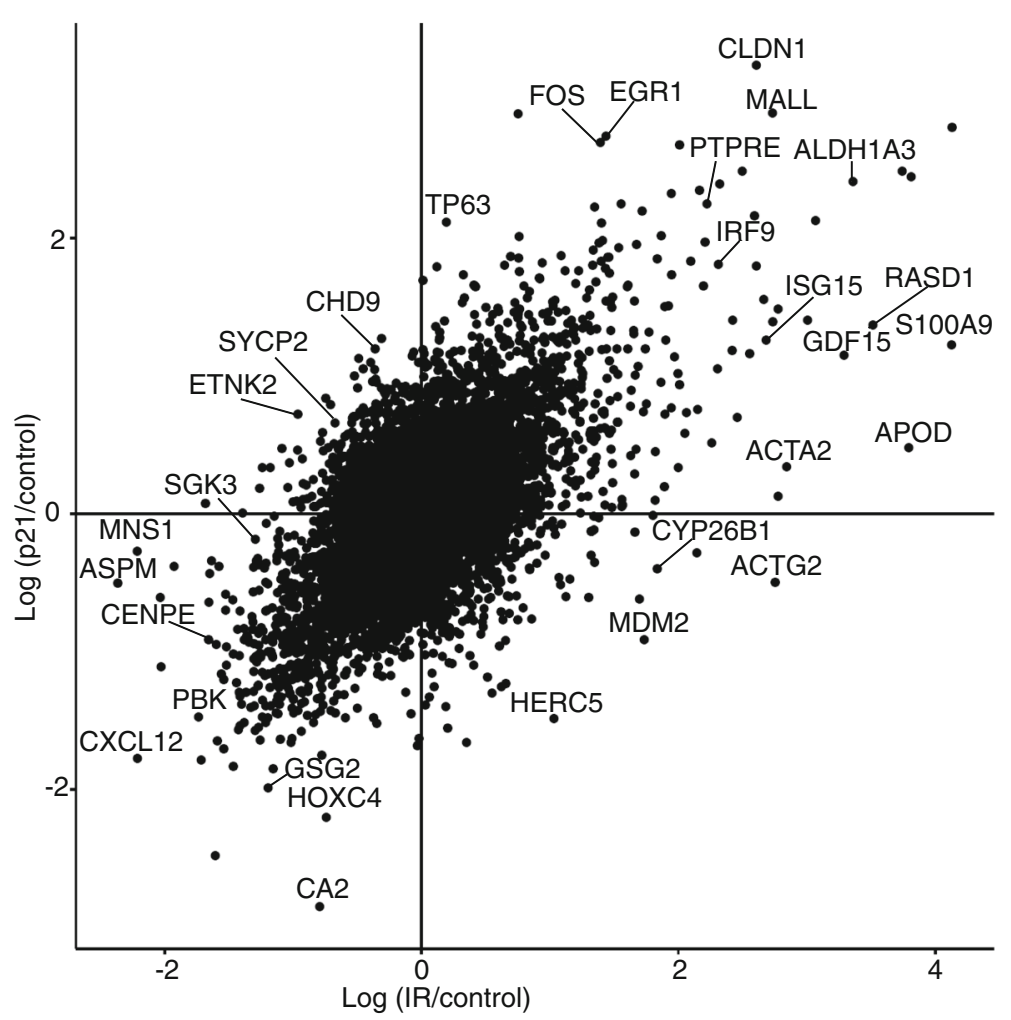

Fig. 2 Radiation- and p21-induced senescence display related gene expression patterns. Plot shows differentially expressed genes detected in radiation-induced and/or p21-induced senescence. Microarray analysis was performed comparing of MCF7 $7^{\text {GFP-IBD }}$ cells, 5 days after 6 Gy irradiation, or MCF $7^{\mathrm{p} 21}$ cells, 5 days after induction with doxycycline, to untreated controls. The relative expression of all detected genes is shown, log transformed. Selected genes are labeled.

downregulated cell cycle genes including transcription factor E2F, cyclins CCNB1 and CCNB2, cyclin-dependent kinase CDK1, replication factor $\mathrm{C}$ components RFC2, RFC3, RFC4, and RFC5, MCM replication factors MCM5 and MCM7, and other regulators of mitosis and cell division including PLK1, CDC25C, and CDC20. IPA analysis also identified three pathways that distinguished radiation-induced senescence from p21-induced senescence, Interferon Signaling, Hepatic Fibrosis/Hepatic Stellate Cell Activation, and Death Receptor Signaling Leading to Apoptosis. In the interferon pathway, genes IFNGR1, STAT1, IRF9, IRF1, IFITM1, IFITM3 were specifically upregulated in the radiation-induced senescent cells (Additional file 3).

Much like the results of IPA analysis, applying DAVID to the 769 shared DEGs yielded 10 KEGG (Kyoto Enrichment of Genes and Genomics) pathways that were linked to DNA replication, cell cycle progression and DNA repair (Table 2).

We also examined enrichment of Gene Ontology (GO) classes with GOrilla and REVIGO. The 769 shared DEGs were ranked from most down-regulated to most up-regulated based on their expression in the radiation-treated cells and input to GOrilla, yielding 18 cellular process $\mathrm{GO}$ terms with cut-off $p$-value $\leq 10^{-7}$ mostly linked to DNA repair, replication and recombination, chromosome segregation and nucleic acid metabolism (Fig. 4).

\section{Analysis of upstream regulators by IPA}

While systems level analysis of the 769 common DEGs identified multiple pathways linked to the proliferative arrest characteristic of senescence, it failed to reveal unanticipated determinants of the senescent cell phenotype. As an alternative strategy, we re-examined the radiation- and p21-associated DEGs by IPA to infer likely upstream regulators (Table 3). Here, analysis is based on comparing an experimental dataset to prior knowledge of transcription factors, microRNAs, kinases and other regulators and their target genes. Regulator "activation" or "inhibition" status was reflected in the sign of the $\mathrm{z}$-score and a $\mathrm{z}$-score threshold of 2 was used for significance. The shared DEGs in both types of senescence identified a common set of predicted upstream regulators including the p21 protein CDKN1A, validating this analysis. Shared regulators included estrogen receptor alpha nuclear 


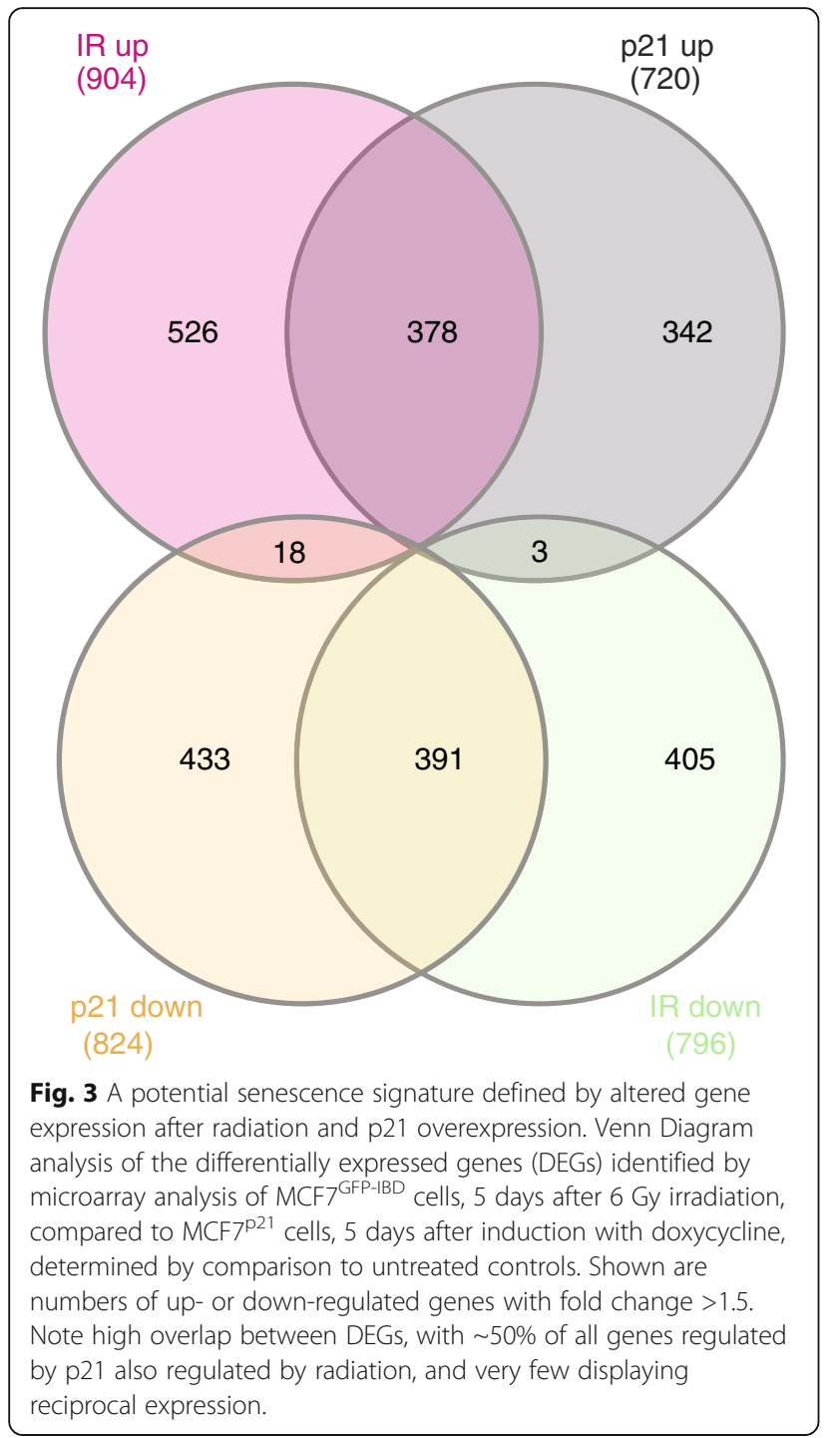

Table 1 Most significant canonical pathways identified by IPA analysis of the shared differentially expressed genes (DEGs)

\begin{tabular}{ll}
\hline Ingenuity canonical pathways & $-\log (p$-value) \\
\hline Role of BRCA1 in DNA Damage Response & 8.54 \\
Role of CHK Proteins in Cell Cycle Checkpoint Control & 8.05 \\
Mitotic Roles of Polo-Like Kinase & 7.54 \\
Cell Cycle: G2/M DNA Damage Checkpoint Regulation & 6.06 \\
Cell Cycle Control of Chromosomal Replication & 5.41 \\
ATM Signaling & 5.22 \\
Mismatch Repair in Eukaryotes & 5.2 \\
Hereditary Breast Cancer Signaling & 4.63 \\
Estrogen-mediated S-phase Entry & 4.58 \\
BER pathway & 3.56 \\
Cyclins and Cell Cycle Regulation & 2.91 \\
B-alanine Degradation I & 2.52 \\
\hline
\end{tabular}

Table 2 Most significant KEGG pathways enriched in the shared differentially expressed genes

\begin{tabular}{lll}
\hline Term & Gene count & $P$-value \\
\hline DNA replication & 17 & $2.00 \mathrm{E}-13$ \\
Cell Cycle & 28 & $4.10 \mathrm{E}-13$ \\
Oocyte meiosis & 18 & $2.10 \mathrm{E}-06$ \\
Base excision repair & 10 & $7.60 \mathrm{E}-06$ \\
Mismatch repair & 8 & $3.00 \mathrm{E}-05$ \\
Nucleotide excision repair & 9 & $4.20 \mathrm{E}-04$ \\
p53 signaling pathway & 10 & $2.00 \mathrm{E}-03$ \\
Progesterone-mediated oocyte maturation & 11 & $2.90 \mathrm{E}-03$ \\
Homologous recombination & 5 & $2.90 \mathrm{E}-02$ \\
Pyrimidine metabolism & 9 & $4.40 \mathrm{E}-02$ \\
\hline
\end{tabular}

hormone receptor ESR1, forkhead box $\mathrm{M} 1 \mathrm{G} 2 / \mathrm{M}$ cell cycle regulatory transcription factor FOXM1, nuclear protein 1 stress response transcriptional regulator NUPR1, and Jarid1B histone H3K4 lysine demethylase KDM5B, each of which has previously been linked to senescence [34-38] but also to diverse other cell responses. In turn, other candidate upstream regulators were identified that were specific to the radiation- or p21-induced senescent samples. Surprisingly, most of the radiation-specific candidate regulators were not DNA damage response factors but linked to other cell signaling pathways.

\section{cmap analysis to identify senescence inducers}

Overall, the results with pathway and upstream regulator analysis failed to confirm that the DEGs shared between radiation- and p21-induced senescence represented a specific signature. As an alternative strategy, we sought to determine if the gene expression pattern represented by the shared DEGs might be able to identify other conditions that induce accelerated senescence. Thus, we queried the Connectivity Map (cmap) database with the 769 shared DEGs toward discovering compounds that induce a similar or reciprocal gene expression pattern. cmap applies a pattern-matching algorithm to compare an input query to a database of microarray analyses of the gene expression effects of treating human cell lines with a diverse set of small molecules. Performing a cmap search based on positive enrichment and limiting analysis to data obtained with MCF7 cells yielded 20 compounds (Table 4). To validate these results, we compared treating $\mathrm{MCF}^{\mathrm{p} 21}$ cells with the 16 commercially available compounds over a range of concentrations to inducing p21 with doxycycline. Following 5 days of incubation, six of the compounds induced characteristic features of senescence including increased cell size, flattened cell shape, and a positive SA- $\beta$-Gal assay (Fig. 5a). The topoisomeraseII poison etoposide $(10 \mu \mathrm{M})$, the anthracycline daunorubicin $(0.1 \mu \mathrm{M})$, the steroidal lactone withaferin $\mathrm{A}(1 \mu \mathrm{M})$, the alpha 


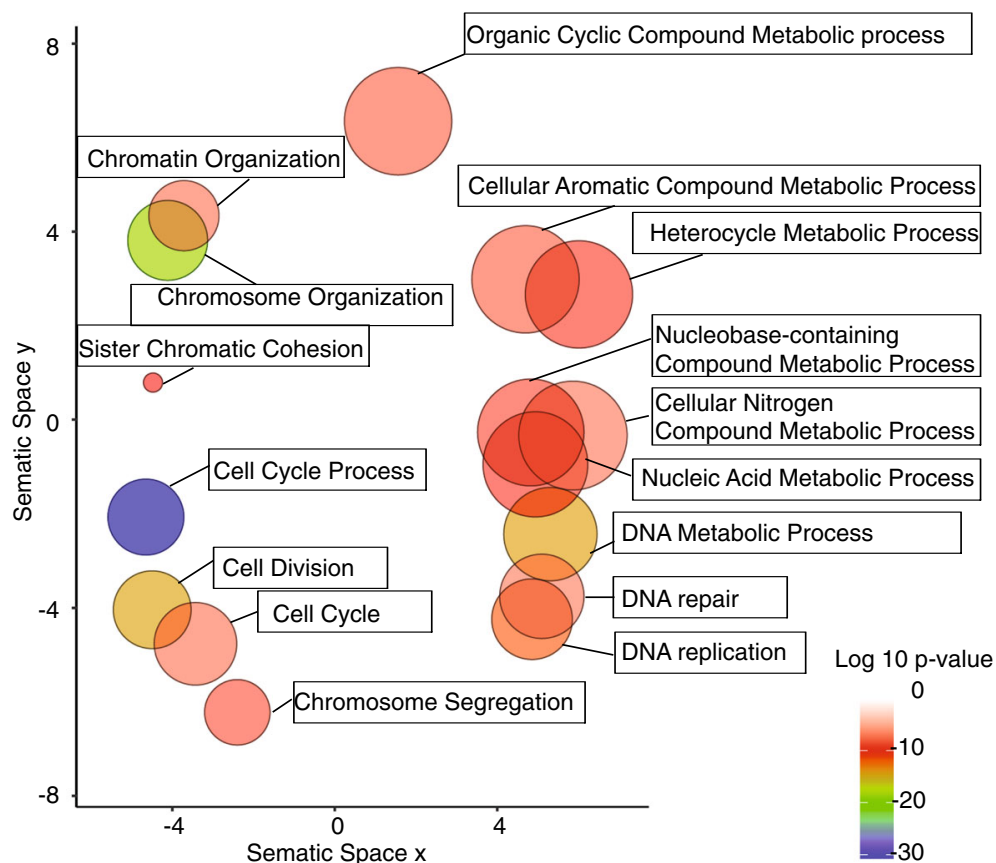

Fig. 4 Gene ontology analysis identifies cell proliferation pathways in the senescence signature. The DEGs shared between radiation- and p21induced senescent cells were subjected to GO enrichment analysis, then summarized and visualized as a scatter plot using the REVIGO Web server. GO terms related to cellular process are represented by circles and are plotted according to semantic similarities to other GO terms (adjoining circles are most closely related). Circle size is proportional to the frequency of the GO term, while color indicates the log $10 p$ value (red higher, blue lower). GO terms with a $\log 10 p$ value of -7 or less for cellular process are labeled.

adrenergic receptor blocker phenoxybenzamine $(50 \mu \mathrm{M})$, the histone deacetylase (HDAC) inhibitor vorinostat (suberoylanilide hydroxamic acid, SAHA, $1 \mu \mathrm{M}$ ), and the selective estrogen receptor degrader fulvestrant $(1 \mu \mathrm{M})$ each scored as a senescence inducer.

Given that two of the small molecule senescence inducers, etoposide and daunorubicin, were well\#characterized genotoxic chemotherapy agents, we treated MCF7 ${ }^{\mathrm{GFP}-\mathrm{IBD}}$ cells with each compound and evaluated formation of DNA damage foci. Along with the two chemotherapy agents, withaferin A and phenoxybenzamine also induced nuclear GFP foci at $24 \mathrm{~h}$ (Fig. 5b), much like radiation. Fulvestrant and vorinostat displayed fewer or no foci, suggesting a DNA damage-independent mechanism of senescence induction.

\section{Discussion}

A wide range of stimuli induce senescence in culture or in tissues, typically via a slow transition where cells initially arrested by a stress response fail to recover and then do not die. While most studied in untransformed, normal cells, senescence can be triggered in immortalized and cancer cells as well. So far no unique regulators have been identified that specify onset of senescence rather than cell death, reversible arrest or proliferation. While most reviews point to unrepaired DNA damage as a common feature underlying senescence, the damage itself appears to be neither necessary nor sufficient. Indeed DNA damage is also cited as a determinant of apoptosis, mitotic catastrophe, and diverse other cellular responses.

Diverse senescence-inducing conditions have been shown to activate expression of the stoichiometric CDK inhibitors (CKIs) p16 ${ }^{\text {INK4A }}$ and/or p $21^{\text {CIP1/WAF1 }}$ [4]. Indeed, p21 is highly induced upon p53 activation, which is considered to be critical for DNA damage induced senescence, and cells with defects in the $\mathrm{p} 53 / \mathrm{p} 21$ and/or $\mathrm{Rb} / \mathrm{p} 16$ pathways are resistant to senescence. In turn, ectopic expression of either p21 or p16 is sufficient to induce the cell cycle arrest and morphological features of senescence [39, 40]. The effects of p21 and p16 may extend beyond their CDK targets, insofar as small-molecule $\mathrm{CDK}$ inhibitors (CKIs) generally fail to induce senescence. Importantly, the CKIs do not induce the characteristic senescent cell secretome, the SASP [14], implicating DNA damage as a specific driver of inflammatory gene expression versus other senescent cell phenotypes.

Building on the pioneering studies that identified common senescence markers such as p21 and/or p16 and SA- $\beta$ Gal, several prior system biology-based 
Table 3 Predicted upstream regulators from IPA analysis

\begin{tabular}{ll}
\hline Upstream regulator & Gene description \\
\hline In IR and p21 & \\
ESR1 & Estrogen receptor \\
FOXM1 & Forkhead Box M1 \\
NUPR1 & Nuclear protein, transcriptional \\
& regulator 1 \\
KDM5B & Chromobox Homolog 5 \\
CDKN1A & Cyclin Dependent Kinase \\
In IR only & Inhibitor 1A \\
CBX5 & \\
AURKB & Chromobox Homolog 5 \\
KIAA1524 & Aurora Kinase B \\
ANXA2 & KIAA1524 \\
MAPK1 & Annexin A2 \\
H2AFY & Mitogen-Activated Protein Kinase 1 \\
EPAS1 & H2A Histone Family Member Y \\
CCL5 & Endothelial PAS Domain Protein 1 \\
ERBB2 & C-C Motif Chemokine Ligand 5 \\
TGFBR2 & Erb-B2 Receptor Tyrosine Kinase 2 \\
AR & Transforming Growth Factor Beta \\
COL18A1 & Receptor 2 \\
EZH2 & Androgen Recptor \\
KLF4 & Collagen Type XVIII Alpha 1 Chain \\
\hline & Enhancer Of Zeste 2 Polycomb \\
& Repressive Complex 2 Subunit \\
& \\
\hline &
\end{tabular}

efforts to discover signatures of senescence have applied transcriptomics or proteomics to examine replicative senescence $[41,42]$ and accelerated senescence [43] in cancer cells and in aging organs of human or animals [8,9]. While these studies have revealed the common pattern of activation of inflammatory pathways linked to the SASP [10, 11, $44,45]$, the gene expression patterns that underlie other characteristic features of senescence have remained largely obscure. We sought to explore whether comparing the transcriptomes of senescent cells induced by DNA damage or CKI overexpression might identify a core senescence gene expression pattern. Thus, we compared the differential gene expression induced in the otherwise immortal breast carcinoma cell line MCF7 $\left(\mathrm{ER}^{+}, \mathrm{INK} 4 \mathrm{~A}^{-}\right.$, Caspase $\left.3^{-}\right)$ by treatment with ionizing radiation or Tet-On inducible expression of p21. Microarray analysis performed 5 days after irradiation or inducing p21 with doxycycline yielded 790 genes, which were differentially expressed under each condition. Of these, 769 (97\%) displayed the same direction of change, suggesting that these shared differentially expressed genes (DEGs) might represent a senescence signature. As expected from the proliferative arrest in senescence, systems level analysis of the shared DEGs identified changes in multiple pathways linked to cell cycle progression and DNA metabolism. Overall though, analysis of pathways failed to detect a specific pattern that might distinguish senescence from other cell stress responses.

Prior studies have revealed a critical role for reactive oxygen species (ROS) in p21-dependent senescence [46], potentially mediating the effects of p21 in part via inducing a DNA damage signal. Treatment with $\mathrm{N}$-acetyl cysteine to reduce ROS could block p21 overexpression from inducing senescence in multiple cell lines, including cancer cells [47]. Nonetheless, our gene expression analysis revealed no elevation of oxidative response genes such as SOD2, CAT, GPX2, or PRDX after p21 induction. In turn, adding $\mathrm{N}$-acetyl cysteine failed to prevent p21 from inducing senescence in the MCF7 cells (data not shown). Although multiple pathways linked to DNA damage response were enriched by the DEGs shared between p21 overexpression and irradiation, examining the specific DEGs that had populated the DDR pathways showed that most were linked to cell cycle progression or arrest rather than DNA repair per se. In turn, the few shared DEGs with an established role in DNA damage repair such as RAD51 and BARD1 displayed decreased expression compared to controls.

As a complementary strategy, we queried IPA to identify potential upstream regulatory factors for the DEGs from the radiation- and p21-induced senescent cells. Among factors common to both conditions were CDKN1A, ESR1, FOXM1, NUPR1, and KDM5B. CDKN1A is p21, validating the approach, and ESR1 is estrogen receptor alpha, likely reflecting our use of $\mathrm{ER}^{+}$MCF7 human mammary carcinoma cells. KDM5B is the Jarid1D H3K4 demethylase, previously linked to senescence via the $\mathrm{Rb}$ pathway [34, 48, 49]. FOXM1 is a proliferation-associated transcription factor [36] which antagonizes senescence. NUPR1 is a chromatin-binding protein that confers stress resistance. NUPR1 was found to modulate K-RAS -induced senescence and regulate genome-wide DNA methylation [35]. While KDM5B, FOXM1 and NUPR1 are clearly involved in other processes, these regulators may well play significant roles in control of senescence. However, a prior analysis comparing replicative senescence and accelerated senescence by Kural et al. [50] identified a distinct group of common transcription factors, consistent with the overall uncertainties of this strategy. 
Table 4 Top 20 hits identified through the Connectivity Map

\begin{tabular}{|c|c|c|c|c|c|c|}
\hline Rank & Name & Mean & $n$ & Enrichment & $P$-value & Specificity \\
\hline 1 & Etoposide & 0.859 & 2 & 0.999 & 0 & 0 \\
\hline 2 & Pyrvinium & 0.832 & 4 & 0.994 & 0 & 0 \\
\hline 3 & Vorinostat & 0.595 & 7 & 0.952 & 0 & 0.1101 \\
\hline 6 & 15-delta prostaglandin & 0.635 & 8 & 0.828 & 0 & 0.0299 \\
\hline 7 & Trichostatin A & 0.519 & 92 & 0.776 & 0 & 0.1137 \\
\hline 8 & Thioridazine & 0.507 & 11 & 0.703 & 0 & 0.0233 \\
\hline 9 & Sirolimus & 0.288 & 25 & 0.631 & 0 & 0.0241 \\
\hline 11 & Puromycin & 0.724 & 2 & 0.996 & 0.00002 & 0.0215 \\
\hline 12 & Phenoxybenzamine & 0.709 & 3 & 0.992 & 0.00002 & 0.0394 \\
\hline 13 & Trifluoperazine & 0.514 & 9 & 0.724 & 0.00002 & 0.0337 \\
\hline 15 & Rottlerin & 0.632 & 3 & 0.972 & 0.00004 & 0.0052 \\
\hline 16 & Fulvestrant & 0.332 & 21 & 0.529 & 0.00004 & 0.0971 \\
\hline 17 & lonomycin & 0.663 & 3 & 0.96 & 0.0001 & 0.0053 \\
\hline 18 & Withaferin A & 0.711 & 2 & 0.986 & 0.00034 & 0.0212 \\
\hline 19 & Daunorubicin & 0.605 & 3 & 0.938 & 0.0004 & 0.034 \\
\hline 20 & Clotrimazole & 0.606 & 3 & 0.937 & 0.0004 & 0.0161 \\
\hline 22 & Hycanthone & 0.658 & 2 & 0.985 & 0.00044 & 0.0209 \\
\hline 24 & Niclosamide & 0.68 & 2 & 0.984 & 0.00048 & 0.0101 \\
\hline 26 & Nortriptyline & 0.673 & 2 & 0.983 & 0.00056 & 0 \\
\hline 28 & Ivermectin & 0.659 & 2 & 0.98 & 0.0007 & 0.0152 \\
\hline
\end{tabular}

Hits are limited to perturbagens examined with MCF7 and that yielded a positive score. Under the name of the perturbagen, all instances (different concentration or batch of the same perturbagen for treatment) are included and the mean is the arithmetic mean of the connectivity scores for those instances. The list is ranked based on ascending order of $p$-value then ascending order of enrichment

As an alternative strategy, we sought a means to determine if recapitulating the pattern of gene expression described by the shared DEGs might be sufficient to induce senescence. Here, we took advantage of the Connectivity Map (cmap) database of transcriptional responses to small molecules [51-53]. By searching a query pattern of gene expression against the cmap library, it is possible to identify existing molecules whose effects phenocopy a physiological response, an approach with broad applications for discovery of chemical probes and drugs. To examine the potential to find senescence inducing agents, we queried the database with the shared DEGs to identify compounds that induce similar gene expression patterns in MCF7 cells. Of the top 20 compounds identified by cmap analysis, we obtained 16 and found that six could indeed induce senescence. Four compounds phenocopied radiation-induced senescence by inducing DNA damage foci after 1 day and then displayed senescent morphology and SA- $\beta-$ Gal activity after 5 days. Two of these agents, the semisynthetic podophyllotoxin derivative etoposide and the natural product anthracycline daunorubicin, are well-characterized genotoxic agents that have long been considered topoisomerase II poisons [54, 55]. Multiple epipodophyllotoxins and anthracyclines are effective senescence inducers in MCF7, validating the cmap screen. The two other hits that induced DNA damage were unanticipated. The natural product steroidal lactone withaferin $\mathrm{A}$ is toxic to tumor cell lines and has been linked to production of reactive oxygen species, providing a potential source of DNA damage. The alpha-adrenergic antagonist phenoxybenzamine has not been linked to DNA damage, but we only observed these effects at $50 \mu \mathrm{M}$, well above the concentrations used in most studies. Considering the DNA damage foci observed with all four of these agents, the most parsimonious explanation may be that any agent that promotes DNA damage foci that persist for a day or more has potential as a senescence inducer. Our prior studies [23, $56,57]$ suggest that such compounds are relatively rare, except among agents that are already known to induce chromosomal DNA damage on their own, such as chemotherapy agents.

Two other agents induced senescence within 5 days, but without dramatically increasing DNA damage foci. Vorinostat (SAHA) is a histone deacetylase (HDAC) inhibitor that inhibits both class I and class II HDACs. SAHA was shown to induce a senescence-like arrest in MCF7 cells [58] which may reflect increased expression of p21 [59]. The last hit, fulvestrant, is a selective 


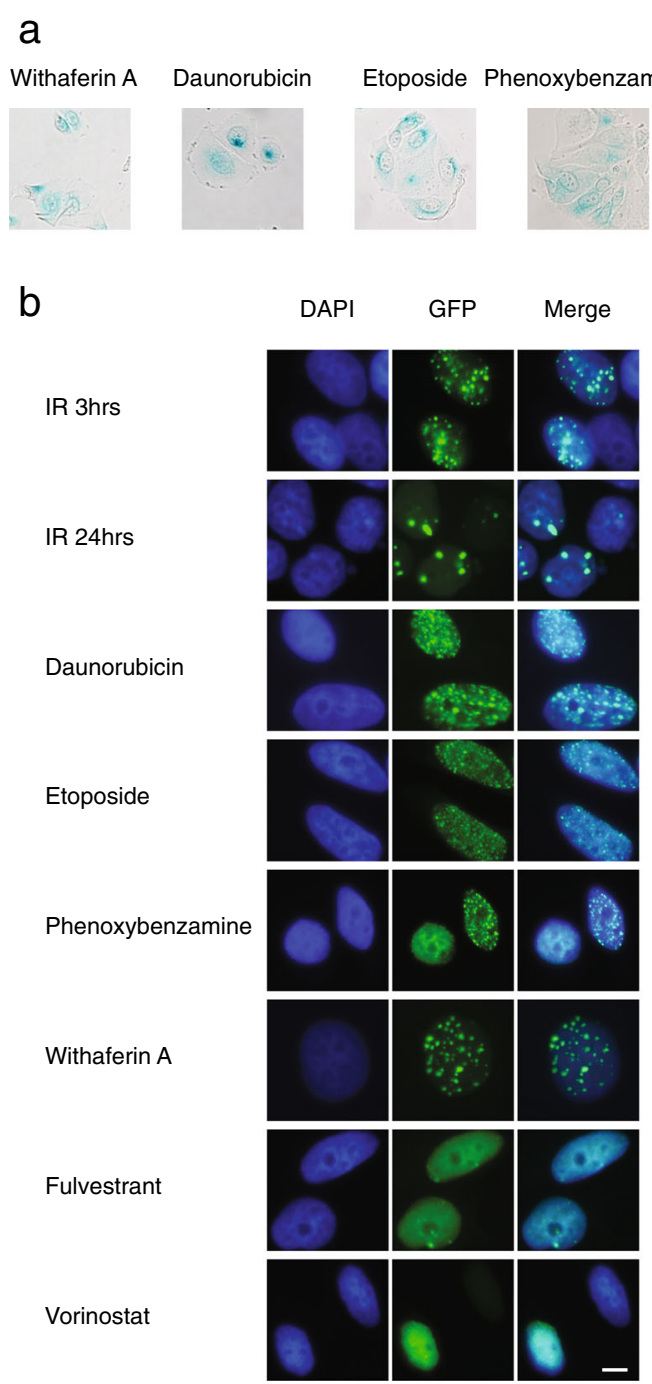

Fig. 5 Senescence phenotypes induced by compounds identified by cmap analysis. a SA- $\beta$-gal assay in MCF $7^{\mathrm{p} 21}$ cells treated with the cmap screen hits, assessed 5 days after adding the indicated compounds. Scale bar $=20 \mu \mathrm{m}$. b GFP-IBD foci in MCF7 GFP-IBD treated with radiation or cmap screen hits. Nuclear GFP foci indicating double strand breaks were observed at $3 \mathrm{~h}$ and $24 \mathrm{~h}$ after 6 Gy irradiation. Treatment with daunorubicin, etoposide, withaferin A, or phenoxybenzamine produced nuclear GFP foci at $24 \mathrm{~h}$. Treatment with fulvestrant or vorinostat failed to induce foci above background. Scale bar $=20 \mu \mathrm{m}$.

estrogen receptor degrader (SERD). Significantly, knockdown of ER in MCF7 cells blocks proliferation in part via derepression of p21 [37], suggesting that fulvestrant may mediate its effect by a similar mechanism. Despite this result and our finding ESR1 as a candidate upstream regulator of senescence, given the wide range of estrogen receptor-dependent genes, fulvestrant is not particularly promising as a chemical probe for senescence.

\section{Conclusions}

Here, we identified differentially expressed genes (DEGs) shared among senescent MCF7 breast carcinoma cells after irradiation or overexpression of the $\mathrm{p} 21^{\mathrm{WAF} 1 / \mathrm{CIP} 1}$ protein and then applied system biology methods to explore the shared DEGs as a candidate senescence signature. The analysis highlighted multiple previously annotated pathways related to cell proliferation, consistent with the cell cycle arrest in senescent cells. A complementary analysis of upstream regulators revealed multiple factors previously implicated in senescence, further validating the strategy. Systems analysis also confirmed that senescence induced by $\mathrm{p} 21$ overexpression lacks the inflammatory signaling prominent in senescence induced by radiation. As a final approach to validation, we queried the cmap database of drug response patterns with the shared DEGs and identified 
multiple compounds that could induce senescence on their own. Taken together, these results suggest that senescence induced by radiation or $\mathrm{p} 21$ expression share a core gene expression pattern linked to key features of the senescent phenotype. The discovery of small molecule senescence inducers by querying cmap with this pattern may point to having identified a signature of cell stress upstream of senescence rather than the cell state per se. Further analysis may identify novel targets to modulate therapy-induced senescence in cancer treatment.

\section{Additional files}

Additional file 1: Python script to calculate the average gene expression. (PY 683 bytes)

Additional file 2: The differentially expressed genes shared between IRand p21-induced senescence. (XLSX 38 kb)

Additional file 3: Differentially expressed genes in interferon signaling pathway in IR-induced senescence. (XLS 28 kb)

\section{Abbreviations}

CKI: CDK inhibitors; cmap: Connectivity Map; DAVID: Database for Annotation, Visualization and Integrated Discovery; DEG: Differentially expressed genes; DSB: Double strand break; GO: Gene Ontology; HDAC: Histone deacetylase; IPA: Ingenuity Pathway Analysis; IR : Ionizing radiation; IRIF: Ionizing radiation induced foci; KEGG: Kyoto Enrichment of Genes and Genomics; ROS: Reactive oxygen species; RS: Replicative senescence; SAM: Significance Analysis of Microarrays; SASP: Senescenceassociated secretory phenotype; SA- $\beta$-Gal: Senescence-associated $\beta$ galactosidase activity; SERD: Selective estrogen receptor degrader; SIPS: Stress-induced premature senescence; TIS: Therapy-induced senescence

\section{Acknowledgements}

This study was supported by NCI R01s CA176843 and CA164492. We thank the University of Chicago Functional Genomics Core Facility for assistance with mRNA microarray analysis and Integrated Microscopy Core Facility for technical support. We thank Dr. Nikolai Khodarev for guidance on the microarray studies, Brian Mitchell for contributing a Python script to sort probes to genes, Yun Feng for transcriptomic and systems analysis, and Rani Hassoun for graphics assistance.

\section{Funding}

These studies were supported by NCI R01s CA176843 and CA164492.

\section{Availability of data and materials}

The dataset in the current study was submitted to GSE and recorded as GSE108895.

\section{Authors' contributions}

SJK supervised the study, DW implemented experiment and performed data analysis, BP participated in data analysis, ST made MCF $7^{\mathrm{p} 21}$ constructs. All authors have read and approved final manuscript.

\section{Ethics approval and consent to participate}

Not applicable.

\section{Consent for publication}

Not applicable.

\section{Competing interests}

The authors declare that they have no competing interests.

\section{Publisher's Note}

Springer Nature remains neutral with regard to jurisdictional claims in published maps and institutional affiliations.

\section{Author details}

${ }^{1}$ Department of Molecular Genetics and Cell Biology and Ludwig Center for Metastasis Research, The University of Chicago, 929 East 57th Street, GCIS W522A, Chicago, IL 60637, USA. ${ }^{2}$ Department of Otolaryngology - Head and Neck Surgery, Feinberg School of Medicine, Northwestern University, Chicago, IL 60611, USA.

Received: 1 June 2018 Accepted: 27 March 2019

Published online: 15 April 2019

\section{References}

1. Hayflick L, Moorhead PS. The serial cultivation of human diploid cell strains. Exp Cell Res. 1961;25(3):585-621.

2. von Zglinicki T. Oxidative stress shortens telomeres. Trends Biochem Sci. 2002;27(7):339-44.

3. Kuilman T, Michaloglou C, Mooi WJ, Peeper DS. The essence of senescence. Genes Dev. 2010;24(22):2463-79.

4. Campisi J. Aging, cellular senescence, and cancer. Annu Rev Physiol. 2013; 75:685.

5. Sharpless NE, Sherr CJ. Forging a signature of in vivo senescence. Nat Rev Cancer. 2015;15(7):397-408

6. Burton DG, Krizhanovsky V. Physiological and pathological consequences of cellular senescence. Cell Mol Life Sci. 2014;71(22):4373-86.

7. Muñoz-Espín D, Cañamero M, Maraver A, Gómez-López G, Contreras J, Murillo-Cuesta S, Rodríguez-Baeza A, Varela-Nieto I, Ruberte J, Collado M. Programmed cell senescence during mammalian embryonic development. Cell. 2013:155(5):1104-18.

8. Wang S, Lu Y, Sun X, Wu D, Fu B, Chen Y, Deng H, Chen X. Identification of common and differential mechanisms of glomerulus and tubule senescence in 24-month-old rats by quantitative LC-MS/MS. Proteomics. 2016;16(20):2706-17.

9. Lu Y, Wang J, Dapeng C, Wu D, Cai G, Chen X. Bioinformatics analysis of proteomics profiles in senescent human primary proximal tubule epithelial cells. BMC Nephrol. 2016;17(1):39

10. Xu J, Patel NH, Saleh T, Cudjoe EK Jr, Alotaibi M, Wu Y, Lima S, Hawkridge AM, Gewirtz DA. Differential radiation sensitivity in p53 wild-type and p53deficient tumor cells associated with senescence but not apoptosis or (nonprotective) autophagy. Radiat Res. 2018;190(5):538-57.

11. Kavanagh E, Lindsay S, Halasz M, Gubbins L, Weiner-Gorzel K, Guang M, McGoldrick A, Collins E, Henry M, Blanco-Fernández A. Protein and chemotherapy profiling of extracellular vesicles harvested from therapeutic induced senescent triple negative breast cancer cells. Oncogenesis. 2017 ; 6(10):e388.

12. Dimri GP, Lee X, Basile G, Acosta M, Scott G, Roskelley C, Medrano EE, Linskens M, Rubelj I, Pereira-Smith O. A biomarker that identifies senescent human cells in culture and in aging skin in vivo. Proc Natl Acad Sci. 1995; 92(20):9363-7.

13. Rodier F, Coppé J-P, Patil CK, Hoeijmakers WA, Muñoz DP, Raza SR, Freund A, Campeau E, Davalos AR, Campisi J. Persistent DNA damage signalling triggers senescence-associated inflammatory cytokine secretion. Nat Cell Biol. 2009:11(8):973-9.

14. Coppé J-P, Rodier F, Patil CK, Freund A, Desprez P-Y, Campisi J. Tumor suppressor and aging biomarker p16INK4a induces cellular senescence without the associated inflammatory secretory phenotype. J Biol Chem. 2011:286(42):36396-403.

15. Freund A, Patil CK, Campisi J. p38MAPK is a novel DNA damage responseindependent regulator of the senescence-associated secretory phenotype. EMBO J. 2011;30(8):1536-48.

16. Xu M, Tchkonia T, Ding H, Ogrodnik M, Lubbers ER, Pirtskhalava T, White TA, Johnson KO, Stout MB, Mezera V. JAK inhibition alleviates the cellular senescence-associated secretory phenotype and frailty in old age. Proc Natl Acad Sci. 2015:112(46):E6301-10.

17. Capell BC, Drake AM, Zhu J, Shah PP, Dou Z, Dorsey J, Simola DF, Donahue G, Sammons M, Rai TS. MLL1 is essential for the senescence-associated secretory phenotype. Genes Dev. 2016;30(3):321-36.

18. Tasdemir N, Banito A, Roe J-S, Alonso-Curbelo D, Camiolo M, Tschaharganeh DF, Huang C-H, Aksoy O, Bolden JE, Chen C-C. BRD4 connects enhancer 
remodeling to senescence immune surveillance. Cancer Discov. 2016;6(6): 612-29.

19. Efimova EV, Takahashi S, Shamsi NA, Wu D, Labay E, Ulanovskaya OA, Weichselbaum RR, Kozmin SA, Kron SJ. Linking cancer metabolism to DNA repair and accelerated senescence. Mol Cancer Res. 2015. https://doi.org/10. 1158/1541-7786.MCR-15-0263.

20. Flor A, Doshi A, Kron S. Modulation of therapy-induced senescence by reactive lipid aldehydes. Cell Death Dis. 2016;2:16045.

21. Gonzalez LC, Ghadaouia S, Martinez A, Rodier F. Premature aging/ senescence in cancer cells facing therapy: good or bad? Biogerontology. 2016;17(1):71-87.

22. Velarde MC, Demaria M, Campisi J. Senescent cells and their secretory phenotype as targets for cancer therapy. Cancer Aging. 2013;38:17-27 Karger Publishers.

23. Labay E, Mauceri HJ, Efimova EV, Flor AC, Sutton HG, Kron SJ, Weichselbaum RR. Repurposing cephalosporin antibiotics as pro-senescent radiosensitizers. Oncotarget. 2016;7(23):33919.

24. Petrova NV, Velichko AK, Razin SV, Kantidze OL. Small molecule compounds that induce cellular senescence. Aging Cell. 2016;15:999.

25. Lamb J, Crawford ED, Peck D, Modell JW, Blat IC, Wrobel MJ, Lerner J, Brunet J-P, Subramanian A, Ross KN. The Connectivity Map: using geneexpression signatures to connect small molecules, genes, and disease. Science. 2006;313(5795):1929-35.

26. Efimova EV, Mauceri HJ, Golden DW, Labay E, Bindokas VP, Darga TE, Chakraborty C, Barreto-Andrade JC, Crawley C, Sutton HG. Poly (ADP-ribose) polymerase inhibitor induces accelerated senescence in irradiated breast cancer cells and tumors. Cancer Res. 2010;70(15):6277-82.

27. Sakaue-Sawano A, Kurokawa H, Morimura T, Hanyu A, Hama H, Osawa H, Kashiwagi S, Fukami K, Miyata T, Miyoshi H. Visualizing spatiotemporal dynamics of multicellular cell-cycle progression. Cell. 2008;132(3):487-98.

28. Cayrol C, Knibiehler M, Ducommun B. p21 binding to PCNA causes G1 and G2 cell cycle arrest in p53-deficient cells. Oncogene. 1998;16(3):311.

29. Tusher VG, Tibshirani R, Chu G. Significance analysis of microarrays applied to the ionizing radiation response. Proc Natl Acad Sci. 2001;98(9):5116-21.

30. Huang DW, Sherman BT, Lempicki RA. Bioinformatics enrichment tools: paths toward the comprehensive functional analysis of large gene lists. Nucleic Acids Res. 2008:37(1):1-13.

31. Kanehisa M, Goto S. KEGG: Kyoto encyclopedia of genes and genomes. Nucleic Acids Res. 2000;28(1):27-30.

32. Eden E, Navon R, Steinfeld I, Lipson D, Yakhini Z. GOrilla: a tool for discovery and visualization of enriched $\mathrm{GO}$ terms in ranked gene lists. BMC Bioinformatics. 2009:10(1):48.

33. Supek F, Bošnjak M, Škunca N, Šmuc T. REVIGO summarizes and visualizes long lists of gene ontology terms. PLoS One. 2011;6(7):e21800.

34. Ohta K, Haraguchi N, Kano Y, Kagawa Y, Konno M, Nishikawa S, Hamabe A, Hasegawa S, Ogawa H, Fukusumi T. Depletion of JARID1B induces cellular senescence in human colorectal cancer. Int J Oncol. 2013;42(4):1212-8.

35. Grasso D, Bintz J, Lomberk G, Molejon MI, Loncle C, Garcia MN, Lopez MB, Urrutia R, lovanna JL. Pivotal role of the chromatin protein Nupr1 in Krasinduced senescence and transformation. Sci Rep. 2015;5:17549.

36. Wierstra I. The transcription factor FOXM1 (Forkhead box M1): proliferationspecific expression, transcription factor function, target genes, mouse models, and normal biological roles. Adv Cancer Res. 2012;118:97-398.

37. Liao XH, Lu DL, Wang N, Liu LY, Wang Y, Li YQ, Yan TB, Sun XG, Hu P, Zhang TC. Estrogen receptor a mediates proliferation of breast cancer MCF-7 cells via a p21/PCNA/E2F1-dependent pathway. FEBS J. 2014;281(3): 927-42.

38. Smirnov A, Panatta E, Lena A, Castiglia D, Di Daniele N, Melino G, Candi E. FOXM1 regulates proliferation, senescence and oxidative stress in keratinocytes and cancer cells. Aging (Albany NY). 2016;8(7):1384.

39. Haferkamp S, Becker TM, Scurr LL, Kefford RF, Rizos H. p16INK4a-induced senescence is disabled by melanoma-associated mutations. Aging Cell. 2008;7(5):733-45.

40. Wang Y, Blandino G, Givol D. Induced p21 waf expression in H1299 cell line promotes cell senescence and protects against cytotoxic effect of radiation and doxorubicin. Oncogene. 1999;18(16):2643.

41. Shelton DN, Chang E, Whittier PS, Choi D, Funk WD. Microarray analysis of replicative senescence. Curr Biol. 1999;9(17):939-45.

42. Purcell M, Kruger A, Tainsky MA. Gene expression profiling of replicative and induced senescence. Cell Cycle. 2014;13(24):3927-37.
43. Mason DX, Jackson TJ, Lin AW. Molecular signature of oncogenic rasinduced senescence. Oncogene. 2004;23(57):9238.

44. Tchkonia T, Zhu Y, Van Deursen J, Campisi J, Kirkland JL. Cellular senescence and the senescent secretory phenotype: therapeutic opportunities. J Clin Invest. 2013;123(3):966-72.

45. Rodier F, Coppé J-P, Patil CK, Hoeijmakers WA, Muñoz DP, Raza SR, Freund A, Campeau E, Davalos AR, Campisi J. Persistent DNA damage signaling triggers senescence-associated inflammatory cytokine secretion. Nat Cell Biol. 2009;11(8):973.

46. Passos JF, Nelson G, Wang C, Richter T, Simillion C, Proctor CJ, Miwa S, Olijslagers S, Hallinan J, Wipat A. Feedback between p21 and reactive oxygen production is necessary for cell senescence. Mol Syst Biol. 2010;6(1):347.

47. Macip S, Igarashi M, Fang L, Chen A, Pan ZQ, Lee SW, Aaronson SA. Inhibition of p21-mediated ROS accumulation can rescue p21-induced senescence. EMBO J. 2002;21(9):2180-8.

48. Chicas A, Kapoor A, Wang X, Aksoy O, Evertts AG, Zhang MQ, Garcia BA, Bernstein E, Lowe SW. H3K4 demethylation by Jarid1a and Jarid1b contributes to retinoblastoma-mediated gene silencing during cellular senescence. Proc Natl Acad Sci. 2012;109(23):8971-6.

49. Nijwening JH, Geutjes E-J, Bernards R, Beijersbergen RL. The histone demethylase Jarid $1 \mathrm{~b}(\mathrm{Kdm} 5 \mathrm{~b})$ is a novel component of the $\mathrm{Rb}$ pathway and associates with E2f-target genes in MEFs during senescence. PLoS One. 2011;6(9):e25235.

50. Kural KC, Tandon N, Skoblov M, Kel-Margoulis OV, Baranova AV. Pathways of aging: comparative analysis of gene signatures in replicative senescence and stress induced premature senescence. BMC Genomics. 2016;17(14):1030.

51. Lamb J. The Connectivity Map: a new tool for biomedical research. Nat Rev Cancer. 2007;7(1):54-60.

52. Qu XA, Rajpal DK. Applications of Connectivity Map in drug discovery and development. Drug Discov Today. 2012;17(23):1289-98.

53. Raghavan R, Hyter S, Pathak HB, Godwin AK, Konecny G, Wang C, Goode EL, Fridley BL. Drug discovery using clinical outcome-based Connectivity Mapping: application to ovarian cancer. BMC Genomics. 2016;17(1):811.

54. Ross W, Rowe T, Glisson B, Yalowich J, Liu L. Role of topoisomerase II in mediating epipodophyllotoxin-induced DNA cleavage. Cancer Res. 1984; 44(12 Part 1):5857-60.

55. Zunino F, Capranico G. DNA topoisomerase II as the primary target of antitumor anthracyclines. Anticancer Drug Des. 1990;5(4):307-17.

56. Labay E, Efimova EV, Quarshie BK, Golden DW, Weichselbaum RR, Kron SJ. lonizing radiation-induced foci persistence screen to discover enhancers of accelerated senescence. Int J High Throughput Screen. 2011;2:1.

57. Efimova EV, Ricco N, Labay E, Mauceri HJ, Flor AC, Ramamurthy A, Sutton HG, Weichselbaum RR, Kron SJ. HMG-CoA reductase inhibition delays DNA repair and promotes senescence after tumor irradiation. In: AACR; 2018.

58. Munster PN, Troso-Sandoval T, Rosen N, Rifkind R, Marks PA, Richon VM. The histone deacetylase inhibitor suberoylanilide hydroxamic acid induces differentiation of human breast cancer cells. Cancer Res. 2001;61(23):8492-7.

59. Huang L, Sowa Y, Sakai T, Pardee AB. Activation of the p21WAF1/CIP1 promoter independent of p53 by the histone deacetylase inhibitor suberoylanilide hydroxamic acid (SAHA) through the Sp1 sites. Oncogene. 2000;19(50):5712.

\section{Ready to submit your research? Choose BMC and benefit from:}

- fast, convenient online submission

- thorough peer review by experienced researchers in your field

- rapid publication on acceptance

- support for research data, including large and complex data types

- gold Open Access which fosters wider collaboration and increased citations

- maximum visibility for your research: over $100 \mathrm{M}$ website views per year

At BMC, research is always in progress.

Learn more biomedcentral.com/submission 